\title{
Thermo-magnetic dependent couplings for quark matter
}

\author{
V. S. Timóteo ${ }^{1, \star}$, R. L. S. Farias ${ }^{2, \star \star}$, S. S. Avancini ${ }^{3, \star \star \star}$, M. B. Pinto ${ }^{3, \star \star \star \star}$ and W. R. Tavares ${ }^{3, \dagger}$ \\ ${ }^{1}$ Faculdade de Tecnologia, Universidade Estadual de Campinas - UNICAMP, Limeira, SP, Brasil \\ ${ }^{2}$ Departamento de Física, Universidade Federal de Santa Maria - UFSM, Santa Maria, RS, Brasil \\ ${ }^{3}$ Departamento de Física, Universidade Federal de Santa Catarina - UFSC, Florianṕolis, SC, Brasil
}

\begin{abstract}
In this work, we improve our model by adding the strange quark and the Polyakov loop. We then build a thermo-magnetic dependence for the $\mathrm{G}$ and $\mathrm{K}$ couplings of the SU(3) PNJL model by fitting lattice QCD calculations for the average and the difference of $u$ and $d$ quark condensates under a strong magnetic field.
\end{abstract}

\section{Introduction}

NJL based interactions have been used as effective models for QCD for quite some time due to their simplicity and clear description of fundamental properties like chiral symmetry breaking and light hadron spectrum [1-4]. With development of lattice techniques, quantities like quark condensates are now calculated directly from QCD so that effective models have now a new reference to constraint their parametrization. If we take a standard parametrization of the PNJL model, with no magnetic field, to calculate the quark condensates and compare to lattice QCD simulations, we can see in Fig. 1 that not only the position of the phase transition is wrong but also its sharpness. This suggests that the effective model needs to be modified in order to describe the behavior of the condensates revealed by lattice QCD calculations. In the following we describe how we construct a thermo-magnetic coupling based on lattice QCD results.

\section{Thermo-magnetic couplings}

In recent letter [5], we presented a an SU(2) NJL model with a coupling which depends on the magnetic field in order to calculate the neutral pion mass at $\mathrm{T}=0$. We also considered the finite temperature case and studied thermodynamic quantities for magnetized quark matter [6] based on a thermomagnetic dependent coupling for the SU(2) NJL model. Here we extend the same scheme to the SU(3) PNJL model in order to obtain temperature and magnetic field dependent couplings $G(e B, T)$ and $K(e B, T)$.

The first attempt to modify the NJL coupling in order to improve the description of lattice QCD calculations was made by Farias et al. [7]. They guessed an analytical formula for the coupling

\footnotetext{
^e-mail: varese@ft.unicamp.br (Grupo de Óptica e Modelagem Numérica - GOMNI)

$\star \star$ e-mail: ricardo.farias@ufsm.edu.br

$\star \star \star$ e-mail: sidney.avancini@fsc.ufsc.br

$\star \star \star \star$ e-mail: marcus.benghi@fsc.ufsc.br

†e-mail: william.tavares@posgrad.ufsc.br
} 


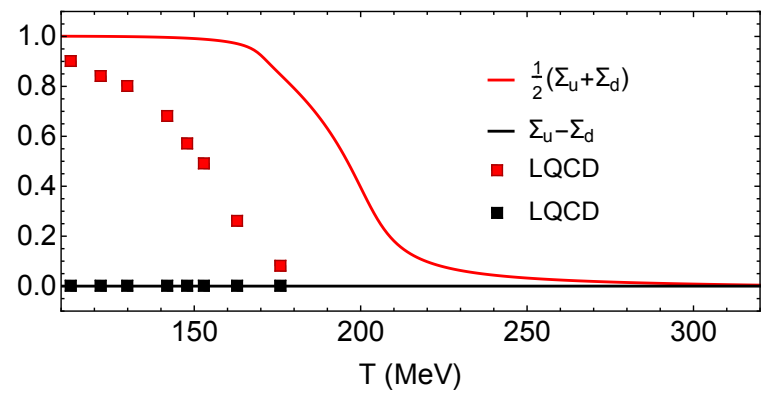

Figure 1. Average and difference of light quark condensates with no magnetic field, $B=0$, from the PNJL model (solid lines) compared to lattice QCD results (squares).

inspired by confinement and showed an improvement in the condensates when compared to lattice results. Later we refined the idea by performing a fit for the NJL coupling to lattice calculations for different temperatures and magnetic fields [6].

For selected values of the magnetic field and temperature given by the lattice QCD collaboration [8-10], we determine the value of couplings $G$ and $K$ that makes the PNJL model to match lattice QCD results for the light quark condensates. Then we make a secondary fit in order to have the couplings at any temperature for any magnetic field.

\section{Numerical results}

We start with the $B=0$ case: in the upper panel of Fig. 2 we show the values of the couplings $G$ (red squares) and $K$ (blue squares) obtained by fitting the condensates shown in the lower panel. It is remarkable that a small linear decrease in both couplings is enough to provide a perfect match between PNJL model and lattice QCD calculations.

Next we look at the case with a constant magnetic background where the PNJL model also fails to describe the behavior of the condensates. In Fig. 3 we show the results of our fit for $e B=0.2 \mathrm{GeV}^{2}$. Again the fit suggests linear behavior for the couplings but with different slope signs before and after the phase transition for both $G$ and $K$.

Finally we apply the thermo-magnetic couplings to the PNJL model in the calculation of thermal susceptibilities and compare to the results obtained with the standard fixed couplings. This is shown in Fig. 4, where the thermal susceptibilities for $B=0$ (left panels) and $e B=0.2 \mathrm{GeV}^{2}$ (right panels) have been computed with the fixed set of couplings (upper panels) and with the modified couplings (lower panels).

As the new couplings are tied to lattice QCD condensates, the PNJL description of the chiral transition becomes perfect and the thermal susceptibilities enhance, sharpen and their peaks shift from $T \sim 200 \mathrm{MeV}$ to $T \sim 160 \mathrm{MeV}$, for both $B=0$ and $e B=0.2 \mathrm{GeV}^{2}$ cases. 

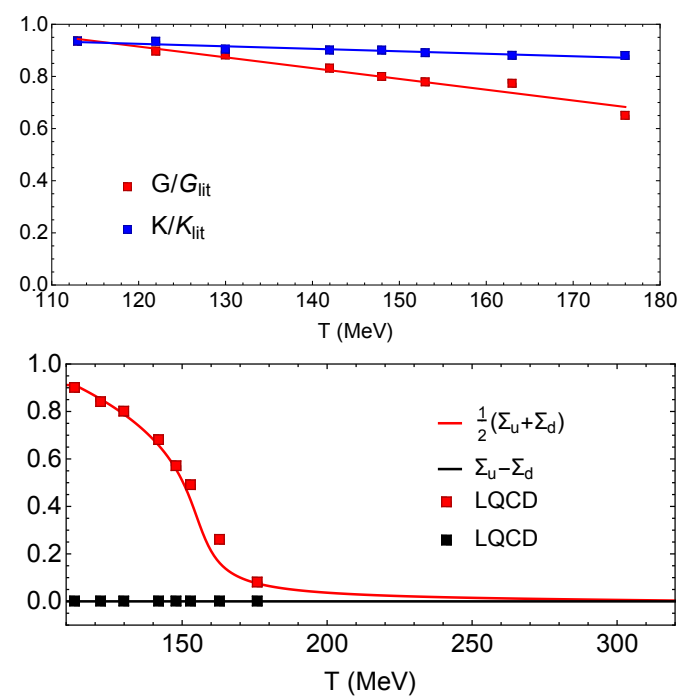

Figure 2. Temperature dependence of PNJL couplings $G$ and $K$ for $B=0$ normalized by the literature values (upper panel) and the quark condensates resulting from the thermo-magnetic couplings (lower panel).
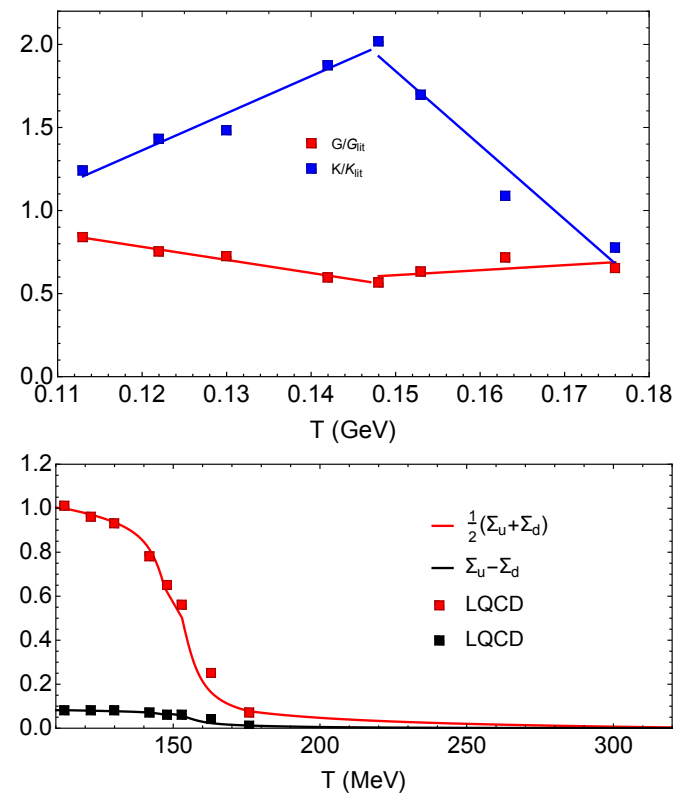

Figure 3. Temperature dependence of PNJL couplings $G$ and $K$ for $e B=0.2 \mathrm{GeV}^{2}$ normalized by the literature values (upper panel) and the quark condensates resulting from the thermo-magnetic couplings (lower panel). 

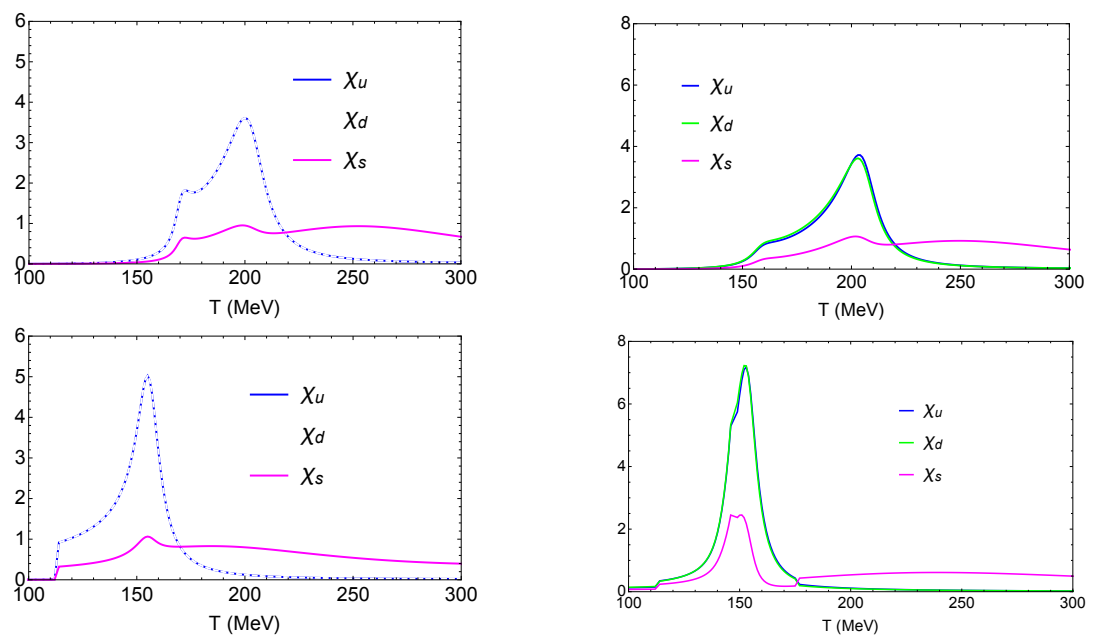

Figure 4. Thermal susceptibility $\chi$ as a function of temperature for $B=0$ (left) and $B=0.2 \mathrm{GeV}^{2}$ (right) with constant couplings (upper panels) and thermo-magnetic couplings (lower panels).

\section{Final remarks}

We propose a set of thermo-magnetic couplings for the PNJL model obtained directly by fitting lattice QCD computations of the quark condensates. For $e B=0$, we find a smooth linear decrease of both $G$ and $K$ while for a finite magnetic background of $e B=0.2 \mathrm{GeV}^{2}$ the couplings also behave linearly, but present change in the slope sign.

\section{Acknowledgements}

We are grateful to FAPESP, CNPq and FAEPEX for financial support.

\section{References}

[1] Y. Nambu, G. Jona-Lasinio, Phys. Rev. 122, 345 (1961).

[2] U. Vogl, W. Weise, Prog. Part. Nucl. Phys. 27, 195 (1991).

[3] S. P. Klevansky, Rev. Mod. Phys. 64, 649 (1992).

[4] M. Buballa, Phys. Rept. 407, 205 (2005).

[5] S. S. Avancini, R. L. S. Farias, M. B. Pinto, W. R. Tavares, V. S. Timóteo, Physics Letters B 767, 247-252 (2017).

[6] R. L. S. Farias, V. S. Timóteo, S. S. Avancini, M. B. Pinto, G. Krein, Eur. Phys. J. A 53, 101 (2017).

[7] R. L. S. Farias, K. P. Gomes, G. Krein, M. B. Pinto, Phys. Rev. C 90, 025203 (2014).

[8] G. S. Bali, F. Bruckmann, G. Endrödi, Z. Fodor, S. D. Katz, A. Schäfer, Phys. Rev. D 86, 071502(R) (2012).

[9] G. S. Bali, F. Bruckmann, G. Endrödi, Z. Fodor, S. D. Katz, S. Krieg, A. Schäfer, K. K. Szabó, JHEP 1202, 044 (2012).

[10] G. S. Bali, F. Bruckmann, G. Endrödi, S. D. Katz, A. Schäfer, JHEP 1408, 177 (2014). 